\title{
Characterization study of As and Se in pyrites from two historic mines in British Columbia
}

\author{
Jocelyn Ross-Lindeman ${ }^{1, *}$, and Dirk Kirste ${ }^{1}$ \\ ${ }^{1}$ Department of Earth Sciences, Simon Fraser University, 8888 University Drive, Burnaby, BC V5A \\ 1S6 Canada
}

\begin{abstract}
Arsenic (As) and selenium (Se) can be toxic if they occur as soluble species at elevated concentrations. One process that can mobilize these elements into the environment is the oxidation of As- and Secontaining pyrites. This study presents the initial mineralogical (XRD, SEM-EDS, LA-ICP-MS, and synchrotron micro-XRF and micro-XANES) characterization of As- and Se-pyrites from two historic mines in British Columbia, the Sullivan Mine and the Sunro Mine. Results show that As occurs in some of the pyrites from the Sullivan Mine; comparison of the micro-XANES measurements to published data suggests As substitutes for sulphur. Selenium is detected in pyrites from the Sunro Mine but this Se is slightly more oxidized than measured in previous studies on Se-pyrite and further investigation of these samples is required. Results from this characterization study will be incorporated into the next phase of research measuring element mobilization after oxidation reactions to identify the effects of As or Se substitution on these reactions.
\end{abstract}

\section{Introduction}

Arsenic (As) and selenium (Se) can be hazardous if the soluble forms of these elements are released into the environment [1]. A major source of this is the oxidation of As- and Secontaining pyrites; this process releases As and Se, which initially occur as insoluble and non-hazardous phases in pyrite, to oxidized and soluble species [1]. Arsenic- and Se-pyrite oxidation has been identified as the initial source of contamination in many locations [e.g. 2-5] although element mobilization can be accelerated by other factors such as irrigation or mining [e.g. 6, 7]. Pyrite oxidation is described as an electrochemical reaction with the reaction at the mineral surface occurring between iron $(\mathrm{Fe})$ in the pyrite and an oxidant, typically ferric iron $[8,9]$; sulphur $(\mathrm{S})$ transfers electrons to $\mathrm{Fe}$ in pyrite as the mineral is a semiconductor and $\mathrm{S}$ has a lower ionization energy [9]. Substitution of elements such as As or Se into pyrite may impact this reaction mechanism. A potential effect is As or Se in pyrite can undergo direct reactions with the oxidant similar to $\mathrm{Fe}$, which has been suggested for elements substituting for Fe [9] and should also be examined for elements substituting for S. As or Se may also be able to transfer electrons to $\mathrm{Fe}$ in pyrite similar to the role of $\mathrm{S}$. A number of studies have examined As-pyrite [e.g. 3, 10-14], but few studies have evaluated As-pyrite oxidation reaction mechanisms and these were focused on specialized

\footnotetext{
*Corresponding author: jocelyn_ross@sfu.ca
} 
oxidants, Se(IV), As(V), and As(III) [12-14]. These studies, however, identified that ferric iron plays a significant role in reactions and that a separate reaction pathway may occur between As and the oxidant [12-14]. Studies on Se-containing pyrite oxidation are even more limited as they are based on field observations not experimental investigations [e.g. 5, 15]. This study summarizes the initial findings from the first phase of research in a largerscale Ph.D. project and describes the characterization of As- and Se- pyrites from two historic mines in British Columbia by scanning electron microscopy energy dispersive spectroscopy (SEM-EDS), X-ray diffraction (XRD), laser ablation inductively coupled plasma mass spectrometry (LA-ICP-MS), and synchrotron micro-X-ray fluorescence (micro-XRF) and micro-X-ray absorption near-edge structure (micro-XANES).

\section{Methods}

Pyritic samples are from the Sullivan Mine and the Sunro Mine [16, 17] and include two hand samples from the Sullivan Mine (SM-1, SM-2) and seven samples total from the Sunro Mine (E-5123175, E-5123177, E-5123179, E-5123180, E-5123174-1 and E5123174-2, E-5123176-1 and E-5123176-2, and E-5123178-1 and E-5123178-2). Samples are all from former mines and thus have been exposed to atmospheric conditions for some time, however, the subsamples used for this study were cut from the interiors of the hand samples. Samples were visually examined and pyritic regions selected for polished section preparation (30 $\mathrm{mm}$ epoxy resin polished sections). Sections selected to best represent the sample mineralogy based on visual examination were also cut and pulverized for XRD analysis. XRD (Bruker D8 Focus X-ray Diffractometer; peak identification, Panalytical HighScore Plus v. 3.0d; Reitveld refinement, Bruker Topas v. 5) and SEM-EDS (TESCAN VEGA 3 SEM with two EDAX Element 30 detectors; TESCAN Integrated Mineral Analyzer [TIMA] platform v. 1.6.22 at a $10 \mu \mathrm{m}$ step size) analyses were conducted at AuTec Innovative Extractive Solutions Ltd. (AuTec; Vancouver, BC). LA-ICP-MS analyses (Thermo Scientific iCAP Qc ICP-MS, New Wave Research [NWR] 213 Nd:YAG laser ablation platform; He carrier gas) were conducted at 4D LABS, Simon Fraser University (SFU; Burnaby, BC). Calibration was on the NIST 610 standard by ablation on a line at a $20 \mu \mathrm{m}$ spot size, a $20 \mathrm{~Hz}$ repetition rate, and a $10 \mu \mathrm{m} / \mathrm{s}$ scan speed at $70 \%$ laser energy (about $22 \pm 2 \mathrm{~J} / \mathrm{cm}^{2}$ ) using element concentrations from the GeoRem database [18]. Ablation on pyrites was on points at a $20 \mu \mathrm{m}$ spot size, a $20 \mathrm{~Hz}$ repetition rate, and a $20 \mathrm{~s}$ dwell time at $35 \%$ laser energy (about $7.5 \pm 2 \mathrm{~J} / \mathrm{cm}^{2}$ ). Ablation started after a 30 second laser warm up; background levels of elements were estimated from measurements collected during the pre-ablation period. Arsenic and Se were measured at a $0.05 \mathrm{~s}$ dwell time and other elements at a $0.02 \mathrm{~s}$ dwell time. The NIST 610 standard, however, was determined to be insufficient for quantification of elements in sulphide minerals and therefore LA-ICPMS measurements were only used to identify if pyrites contain As or Se above background levels. Synchrotron micro-XRF and micro-XANES measurements were conducted at the 20-ID-B beamline at the Advanced Photon Source synchrotron (APS; Argonne, IL) with a $2 \mu \mathrm{m}$ beam size and 4 element Vortex detector for fluorescence measurements [19]. 


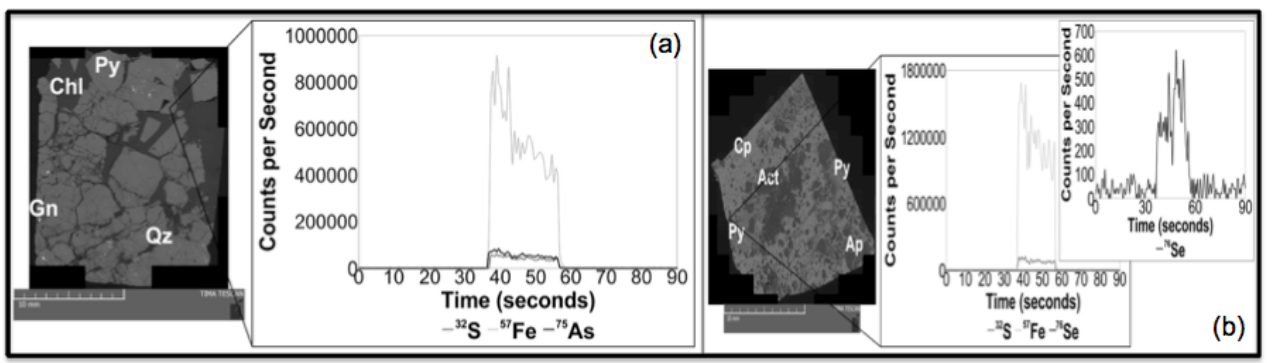

Fig. 1. SEM images and LA-ICP-MS measurements of a polished section from (a) SM-1 (Sullivan Mine) showing As in pyrite and (b) E-5123180 (Sunro Mine) showing Se in pyrite. The LA-ICP-MS measurement of $\mathrm{Se}\left({ }^{76} \mathrm{Se}\right)$ is shown in the inset as Se concentrations are much lower than $\mathrm{Fe}$ and $\mathrm{S}$ $(\mathrm{act}=$ actinolite, $\mathrm{ap}=$ apatite, $\mathrm{cp}=$ chalcopyrite, $\mathrm{chl}=$ chlorite, $\mathrm{gn}=$ galena, $\mathrm{py}=$ pyrite, $\mathrm{qz}=$ quartz $)$.

\section{Results and Discussion}

Modal mineralogy of the Sullivan Mine and Sunro Mine samples was determined from the TIMA SEM measurements and refined using the XRD measurements. SEM analysis included mapping of each polished section; the maps were then used to locate pyrite grains for LA-ICP-MS and synchrotron measurements. From the LA-ICP-MS measurements, pyrites in the SM-1 sample show As concentrations above background; Figure 1a illustrates an SEM image of a polished section from SM-1 and ablation of ${ }^{32} \mathrm{~S},{ }^{57} \mathrm{Fe}$, and ${ }^{75} \mathrm{As}$ in pyrite. Pyrites from the SM-2 sample do not contain As above background. Selenium is not detected (relative to background levels) in any of the Sullivan Mine pyrites but varying concentrations of other elements ( $\mathrm{Co}, \mathrm{Cu}, \mathrm{Zn}, \mathrm{Sb}, \mathrm{Pb}, \pm \mathrm{Bi}$ ) are detected (data not shown). Selenium is detected in pyrites from the Sunro Mine (Fig. 1b). Analyses of these pyrites also show elevated concentrations of other elements ( $\mathrm{Co}, \mathrm{Ni}, \mathrm{Zn}, \mathrm{Cu}, \pm \mathrm{Ag})$ but $\mathrm{As}$ is not detected (data not shown). Synchrotron micro-XRF measurements show that As can be zoned in the SM-1 pyrites (Fig. 2a). Arsenic occurs in a reduced oxidation state, likely the 1 oxidation state, suggesting substitution for S; micro-XANES spectra for As in pyrite show a white line position (large peak) at about $11867 \mathrm{eV}$ calibrated relative to the gold (Au) L3 edge for $\mathrm{Au}$ foil at $11919.4 \mathrm{eV}$ (Fig. 2a), which is similar to XANES measurements of As(-I) in arsenopyrite (Fig. 2a) and As-pyrite from the literature [e.g. 20, 21]. Some oxidation to $\mathrm{As}(\mathrm{V})$ is observed as indicated by a small peak around $11874 \mathrm{eV}$, the position for As(V) bonded to oxygen [e.g. 22, 23] (Fig. 2a), but this may be due to surface oxidation. Selenium is heterogeneously distributed in pyrites in the Sunro Mine samples (Fig. 2b). Selenium in these pyrites is more oxidized than has been measured in previous studies on Se-containing pyrite [e.g. 5, 15] and is shown to occur at a higher oxidation state than elemental $\mathrm{Se}(\mathrm{Se}(0))$ with a white line position (strong peak) about 0.4 $\mathrm{eV}$ higher than $\mathrm{Se}(0)$ (Fig. 2b). This oxidation state for Se, unlike for As in the SM-1 pyrites, may not be from surface oxidation because the XANES spectra does not match published XANES measurements for either of the oxidized Se species, Se(IV) or Se(VI) [24]. The origins of the small positive edge shift for Se-pyrite versus $\operatorname{Se}(0)$ are not clear, but previous research suggests that the behaviour of Se in pyrite is controlled by the conditions under which pyrite formed with both reduced $\mathrm{Se}$ and $\mathrm{Se}(0)$ possible [25]. The different polymorphs of $\mathrm{Se}(0)$ or differences in the coordination environment of Se (e.g. Se-S bonding) can also result in small energy shifts [26]. 


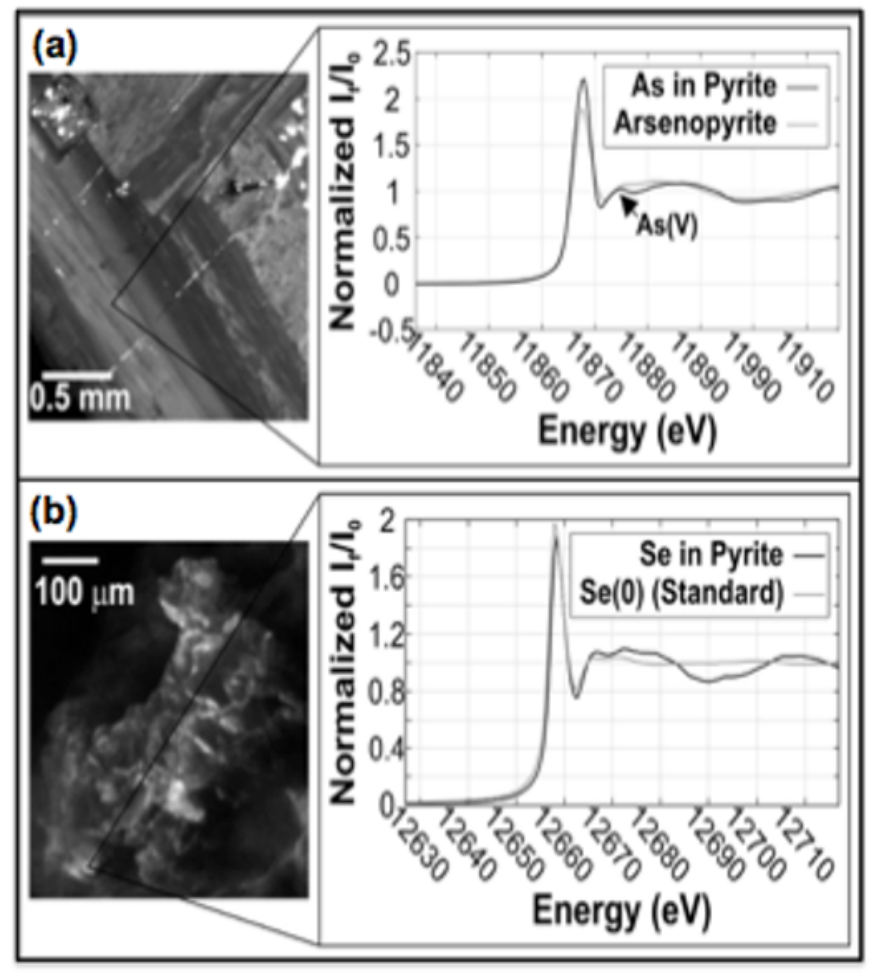

Fig. 2. (a) Micro-XRF map of a pyrite in SM-1 showing As zoning and micro-XANES measurement of As in pyrite. As likely occurs in the -1 oxidation state from comparisons to previous studies [e.g. 20, 21] and to arsenopyrite (As standards library, APS Sector 20, 20-BM, Au foil reference). A small peak around $11874 \mathrm{eV}$ suggests oxidation to $\mathrm{As}(\mathrm{V})$ as it occurs at the position for As(V) bonded to oxygen [e.g. 22, 23]. (b) Micro-XRF map of a pyrite in E-5123180 and micro-XANES measurement of $\mathrm{Se}$ in pyrite. Se in pyrite is slightly more oxidized than $\mathrm{Se}(0)$ as the spectra is shifted to a slightly higher energy than the $\operatorname{Se}(0)$ standard. The maps show the ratio of As or Se fluorescence energy to incident energy $\left(\mathrm{I}_{\mathrm{f}} / \mathrm{I}_{0}\right.$; As $\mathrm{I}_{0}=13100 \mathrm{eV}$ and $\left.\mathrm{Se} \mathrm{I}_{0}=12800 \mathrm{eV}\right)$ with lighter grey colours indicating higher As or Se concentrations (maps generated using ImageJ [27] and the APS Sector 20 inputoutput plugin). Micro-XANES data were processed using Athena [28]. APS Sector 20 As standards and ImageJ plugin from https://s20.xray.aps.anl.gov.

\section{Future Work}

Batch oxidation experiments will be conducted on these pyrites and the concentrations, oxidation states, and rates of As and Se released from pyrite using ferric iron as the oxidant will be measured to better understand the effects of As or Se substitution on oxidation reaction mechanisms. Analyses of the supernatant will measure release of As and Se (rates, oxidation states, concentrations) to determine if the concentrations of the elements mobilized are proportional to their concentrations in pyrite to assess if direct reactions may be occurring between the oxidant and the substituted elements. Analyses of the oxidized pyrites will examine the changes in the oxidation states of As and Se compared to unaltered pyrite to examine electron transfer and reaction pathways.

This research contributes to Ms. Ross-Lindeman's Ph.D. and is funded by SFU (C.D. Nelson Entrance Scholarship, Provost Prize of Distinction). Sullivan Mine samples are from Dr. Diana Allen (Department of Earth Sciences, SFU). Sunro Mine samples are generously provided by New Sunro 
Copper Ltd. We greatly appreciate the in-kind analytical support from AuTec for XRD and SEM analyses. APS Sector 20 operations are supported by the US Department of Energy and the Canadian Light Source (CLS); we acknowledge the receipt of support from the CLSI Graduate and PostDoctoral Student Travel Support Program. This research has benefited from discussions with Ms. Ross-Lindeman's supervisory committee, Dr. Robert Gordon (Department of Physics, SFU) and Dr. Daniel Marshall (Department of Earth Sciences, SFU). We also extend our thanks to the two anonymous reviewers for their insightful comments.

\section{References}

1. J.A. Plant, et al., Treatise on Geochemistry (2 ${ }^{\text {nd }}$ Ed), Environmental Geochemistry, 11, 13-57 (2014)

2. G.B. Dreher, R.B. Finkelman, Env Geol Water Sci, 19, 155-167 (1992)

3. K.S. Savage, et al., Appl Geochem, 15, 1219-1244 (2000)

4. E. Pili, et al., J Hazard Mat, 262, 887-895 (2013)

5. M.J. Hendry, et al., Env Sci Technol, 49, 8228-8236 (2015)

6. T.S. Presser, H.M. Ohlendorf, Env Manag, 11, 805-821 (1987)

7. Y. Cai, et al., Appl Geochem, 84, 254-261 (2017)

8. P.C. Singer, W. Stumm, Science, 167, 1121-1123 (1970)

9. J.D. Rimstidt, D.J. Vaughan, Geochim Cosmochim Acta, 67, 873-880 (2003)

10. A. Kolker, F.E. Huggins, Appl Geochem, 22, 778-787 (2007)

11. S. Lehner, K. Savage, Geochim Cosmochim Acta, 72, 1788-1800 (2008)

12. G. Qiu, et al., Geochim Cosmochim Acta, 217, 306-319 (2017)

13. G. Qiu, et al., Geochim Cosmochim Acta, 228, 205-219 (2018)

14. M. Kang, et al., Appl Geochem, 47, 130-140 (2014)

15. A. Matamoros-Veloza, et al., Env Sci Technol, 48, 8972-8979 (2014)

16. V.G. Ethier, et al., Econ Geol, 71, 1570-1588 (1976)

17. B. Northcote, Exploration and Mining in British Columbia, 2016, British Columbia Geological Survey, Information Circular 2017-1, 133-147 (2017)

18. K.P. Jochum, et al., Geostand Geoanalytical Res, 29, 333-338 (2005)

19. S.M. Heald, et al., Nucl Instrum Meth A, 582, 215-217 (2007)

20. W. Zhu, et al., Geochim Cosmochim Acta, 72, 5243-5250 (2008)

21. J.M. Stromberg, et al., Ore Geol Rev, 104, 589-602 (2019)

22. B.C. Bostick, et al., Env Sci Technol, 38, 3299-3304 (2004)

23. P.G. Smith, et al., Env Sci Technol, 39, 248-254 (2005)

24. I.J. Pickering, et al., Env Sci Technol, 29, 2456-2459 (1995)

25. A. Diener, et al., J Contam Hydrol, 133, 30-39 (2012)

26. G. Sarret, et al., Appl Env Microb, 71, 2331-2337 (2005)

27. C.A. Schneider, et al., Nat Methods, 9, 671-675 (2012)

28. B. Ravel, M. Newville, J Synchrotron Radiat, 12, 537-541 (2005) 